\title{
New ephemeris of the ADC source 2A 1822-371: a stable orbital-period derivative over 30 years
}

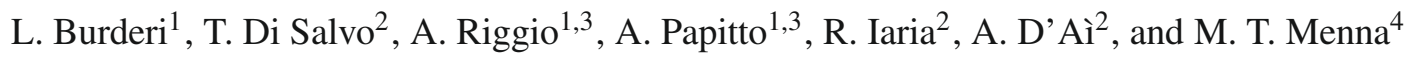 \\ 1 Dipartimento di Fisica, Università degli Studi di Cagliari, SP Monserrato-Sestu, KM 0.7, Monserrato 09042, Italy \\ e-mail: burderi@dsf.unica.it \\ 2 Dipartimento di Scienze Fisiche ed Astronomiche, Università di Palermo, via Archirafi 36, Palermo 90123, Italy \\ e-mail: disalvo@fisica.unipa.it \\ 3 INAF Osservatorio Astronomico di Cagliari, Loc. Poggio dei Pini, Strada 54, 09012 Capoterra (CA), Italy \\ ${ }^{4}$ INAF Osservatorio Astronomico di Roma, via Frascati 33, Monteporzio Catone 00040, Italy
}

Received 13 July 2009 / Accepted 4 March 2010

\section{ABSTRACT}

\begin{abstract}
We report on a timing of the eclipse arrival times of the low mass X-ray binary and X-ray pulsar 2A 1822-371 performed using all available observations of the Proportional Counter Array on board the Rossi X-ray Timing Explorer, XMM-Newton pn, and Chandra. These observations span the years from 1996 to 2008. Combining these eclipse arrival time measurements with those already available covering the period from 1977 to 1996, we obtain an orbital solution valid for more than thirty years. The time delays calculated with respect to a constant orbital period model show a clear parabolic trend, implying that the orbital period in this source constantly increases with time at a rate $\dot{P}_{\text {orb }}=1.50(7) \times 10^{-10} \mathrm{~s} / \mathrm{s}$. This is 3 orders of magnitude larger than what is expected from conservative mass transfer driven by magnetic braking and gravitational radiation. From the conservation of the angular momentum of the system we find that to explain the high and positive value of the orbital period derivative the mass transfer rate must not be less than 3 times the Eddington limit for a neutron star, suggesting that the mass transfer has to be partially non-conservative. With the hypothesis that the neutron star accretes at the Eddington limit we find a consistent solution in which at least $70 \%$ of the transferred mass has to be expelled from the system.
\end{abstract}

Key words. eclipses - ephemerides - pulsars: individual: 2A 1822-371 - X-rays: binaries - stars: winds, outflows accretion, accretion disks

\section{Introduction}

The source 2A 1822-371 is a well-known low mass X-ray binary (hereafter LMXB) seen almost edge-on with an inclination angle of $i \sim 85^{\circ}$ (Hellier \& Mason 1989). The observed average unabsorbed flux of the source in the $0.1-100 \mathrm{keV}$ energy range is $\sim 1.5 \times 10^{-9} \mathrm{erg} \mathrm{cm}^{-2} \mathrm{~s}^{-1}$ (Iaria et al. 2001). This corresponds to an unabsorbed luminosity of $\sim 1.2 \times 10^{36} \mathrm{erg} \mathrm{s}^{-1}$, adopting a distance of $2.5 \mathrm{kpc}$ (Mason \& Cordova 1982). However, it has been noted (Parmar et al. 2000) that the mean ratio of the X-ray over optical luminosity, $L_{\mathrm{X}} / L_{\mathrm{opt}}$, for $2 \mathrm{~A} 1822-371$ is about 20 , while the average value for LMXBs is about 500 (van Paradijs $\&$ McClintock 1994). This would imply an unobscured X-ray luminosity as high as $3 \times 10^{37} \mathrm{erg} / \mathrm{s}$ for the assumed distance of $2.5 \mathrm{kpc}$. The apparent low luminosity of the source has therefore to be ascribed to the high inclination of the system with respect to the line of sight. Indeed, the light curve of 2A 1822-371 shows both dips and eclipses of the X-ray source by the companion star. The partial nature of the eclipse indicates that the X-ray emitting region is extended and that the observed X-rays are scattered in an accretion disk corona (ADC, White et al. 1981). The X-ray light curve shows clear signs of orbital modulation with a binary orbital period of $5.57 \mathrm{~h}$. This X-ray modulation is probably caused by the obscuration of the ADC by the thick rim of an accretion disk. The orbital period has been measured from eclipse timing to increase gradually (Hellier et al. 1990). Parmar et al. (2000) gave the best ephemeris of this source before this work. In particular they found a significant positive orbital period derivative of $\dot{P}_{\text {orb }}=1.78 \times 10^{-10} \mathrm{~s} / \mathrm{s}$.

Jonker \& van der Klis (2001) reported on the discovery of $0.59 \mathrm{~s}$ X-ray pulsations in this source in an RXTE observation performed in 1998. The timing analysis of the pulse arrival times indicates a circular orbit with an eccentricity $e<0.03(95 \%$ c.l.) and an $a \sin i$ for the neutron star of 1.006(5) lt-s, implying a mass function of $(2.03 \pm 0.03) \times 10^{-2} M_{\odot}$. The comparison between the pulse period measured by RXTE in 1996 and 1998 also indicates that the neutron star in this system is spinning up at a rate of $\dot{P}=(-2.85 \pm 0.04) \times 10^{-12} \mathrm{~s} / \mathrm{s}$. Jonker $\&$ van der Klis (2001) inferred a bolometric X-ray luminosity of about $(2-4) \times 10^{37} \mathrm{erg} / \mathrm{s}$ assuming a magnetic field of $(1-5) \times 10^{12}$ Gauss. From spectroscopic measurements of the radial velocity curve of the companion, Jonker et al. (2003) derived a lower limit to the mass of the neutron star and to that of the companion star of $0.97 \pm 0.24$ and $0.33 \pm 0.05 M_{\odot}$, respectively $(1 \sigma$, including uncertainties in the inclination), and an accurate estimate of the system inclination angle, $i=82^{\circ} .5$.

In this paper we report on the analysis of X-ray observations of 2A 1822-371 performed from 1996 to 2008 by RXTE, $X M M-N e w t o n$, and Chandra with the aim to derive eclipse arrival times and to improve the orbital ephemeris. We confirm with higher precision and over a much larger time span (about 31 years) the ephemeris found by Parmar et al. (2000). In particular we find that the orbital period derivative has remained constant during the last 30 years. Finally we discuss the implications 
Table 1. New X-ray eclipse times for 2A 1822-371.

\begin{tabular}{lllll}
\hline \hline$T_{\text {ecl }}($ MJD) & Error & Cycle & Satellite (ObsID) & $T_{\text {start }}-T_{\text {stop }}(\mathrm{MJD})$ \\
\hline 50352.10094 & 0.00047 & 20410 & RXTE (P10115) & $50352.34-50353.44$ \\
50992.0857 & 0.0023 & 23167 & RXTE (P30060) & $50992.81-51019.65$ \\
51975.01132 & 0.00031 & 27402 & RXTE (P50048) & $51975.44-52101.48$ \\
52432.03655 & 0.00030 & 29371 & RXTE (P70036/37) & $52432.37-52435.77$ \\
52487.97497 & 0.00038 & 29612 & RXTE (P70037) & $52488.36-52504.01$ \\
52519.07766 & 0.00085 & 29746 & RXTE (P70037) & $52519.15-52547.87$ \\
52882.09667 & 0.00037 & 31310 & RXTE (P70037) & $52882.02-52885.21$ \\
51975.06934 & 0.00056 & 27402 & XMM-Newton (230101) & $51975.55-51976.15$ \\
51779.6317 & 0.0019 & 26560 & Chandra (671) & $51779.70-51780.16$ \\
54607.19592 & 0.00056 & 38742 & Chandra (9076/9858) & $54606.96-54610.52$ \\
\hline
\end{tabular}

Notes. Uncertainties are calculated as described in the text. $T_{\text {start }}-T_{\text {stop }}$ indicates the time interval over which a folding of the orbital light curve was performed to derive the time of eclipse.

of a high and positive value of the orbital period derivative on the mass transfer rate and secular evolution of this source.

\section{Timing analysis and results}

We analysed all available X-ray observations of 2A 1822-371 performed over the period from 1996 to 2008. In particular we used observations from the PCA on board RXTE performed in 1996 (P10115), 1998 (P30060), 2001 (P50048), 2002 (P70036), 2002-2003 (P70037), one observation from XMM-Newton performed in 2001 (Obs ID: 0111230101 and 0111230201), and two Chandra observations performed in 2000 (Obs ID: 671) and in 2008 (Obs ID: 9076 and 9858), respectively. The arrival times of all events were referred to the solar system barycenter, using as the best estimate for the source coordinates those derived from the 2008 Chandra observations (RA: 1825 46.81, Dec: -37 06 18.5, uncertainty: $\left.0.6^{\prime \prime}\right)$.

The typical eclipse duration is around $2.2 \mathrm{ks}$, which corresponds to $10 \%$ of the binary orbital period. In order to improve the statistics for the measure of the eclipse epochs and to have the possibility of fitting a complete orbital light curve we decided to perform a folding of these data using the known binary orbital period of the source, after verifying that this folding does not affect the results reported here in any case. Folding the data is not an important issue for the two Chandra observations and the XMM observation, where just one or two consecutive eclipses are observed. But it is important for the RXTE observations, because these are short and sparse, and also because the RXTE observations are continuously interrupted by the Earth occultation at every RXTE orbit (lasting approximately $1.5 \mathrm{~h}$ ). In this case the folding is required to sample a complete orbital light curve from the source, because this is important for a meaningful fitting of the eclipse. For each of these observations we hence folded the data using the local orbital period as derived from the ephemeris published by Parmar et al. (2000). The 2002-2003 RXTE dataset (P70036 and P70037) was long enough and we decided to divide it into the following four periods: i) 2002 June 7-10; ii) 2002 August 2-18; iii) 2002 September 2-30; and iv) 2003 August $31-$ September 3. In this way we obtained a total of 10 orbital light curves in which the eclipses were clearly visible (see Table 1 for details on the used observations).

We then fitted these orbital light curves to derive eclipse arrival times with the procedure described below. Because the eclipses are asymmetrical and partial, the exact eclipse centroid times crucially depend on the model adopted to describe their shape as well as the variable continuum they are superimposed on. In order to be conservative in our estimates, we then decided to fit the folded light curves using 10 different models. The first model is that used by Parmar et al. (2000) consisting of a Gaussian and a constant fitted on a phase interval of 0.1 around the eclipse. The second and third models consist again of a Gaussian and a constant plus a linear term (second model) and a linear and quadratic term (third model) fitted on a phase interval of 0.3 around the eclipse. The fourth model is as the third model plus a cubic term fitted on a phase interval of 0.4 around the eclipse. The fifth model consists of a Gaussian and a constant plus a sinusoid of period fixed to the orbital period fitted on the whole $0-1$ phase interval. The models from the sixth one to the tenth one are as the fifth model, plus from 2 to 6 sinusoids with periods fixed to $1 / 2$ up to $1 / 6$ of the orbital period, respectively. The addition of higher harmonic components was required to better describe the overall orbital light curve shape, which differs from a pure sinusoid. We restricted our fitting to the first six harmonics because the addition of higher harmonic components was not statistically significant based on an F-test.

Thus we obtained 10 eclipse arrival times (each corresponding to one of the models described above) for each orbital light curve. The final eclipse arrival time for each orbital light curve was chosen to be the average of these 10 values, and the associated uncertainty was chosen to be half of the maximum range spanned by these values ( $1 \sigma$ error included). The uncertainty derived in this way fully takes into account significant discrepancies among the different eclipse arrival times found with a particular model to describe the eclipse and the orbital modulation. The obtained values of the eclipse epochs for each of the 10 orbital light curves and the relative uncertainties are reported in Table 1.

We then computed the eclipse time delays by subtracting from our measures the eclipse arrival times predicted by a constant orbital period model adopting the orbital period, $P_{\text {orb } 0}$, and the reference time, $T_{0}^{\mathrm{e}}$, given by Parmar et al. (2000). These time delays were plotted versus the orbital cycle number $N$. The integer $N$ is the exact number of orbital cycles elapsed since $T_{0}^{\text {e }}$; i.e., $N$ is the closest integer to $\left(T_{N}^{\mathrm{e}}-T_{0}^{\mathrm{e}}\right) / P_{\text {orb } 0}$ under the assumption that $\left|T_{N}^{\mathrm{e}}-\left(T_{0}^{\mathrm{e}}+N P_{\text {orb } 0}\right)\right| \ll P_{\text {orb } 0}$ that we have verified a posteriori. These results are shown in Fig. 1 together with all delays computed from previously available eclipse times, namely those given by Hellier et al. (1990) and by Parmar et al. (2000), respectively.

These points show a clear parabolic trend that we fitted to the equation

$\delta T_{N}^{\mathrm{e}}=\delta T_{0}^{\mathrm{e}}+\delta P_{\text {orb } 0} \times N+(1 / 2) \dot{P}_{\text {orb }} P_{\text {orb } 0} \times N^{2}$, 


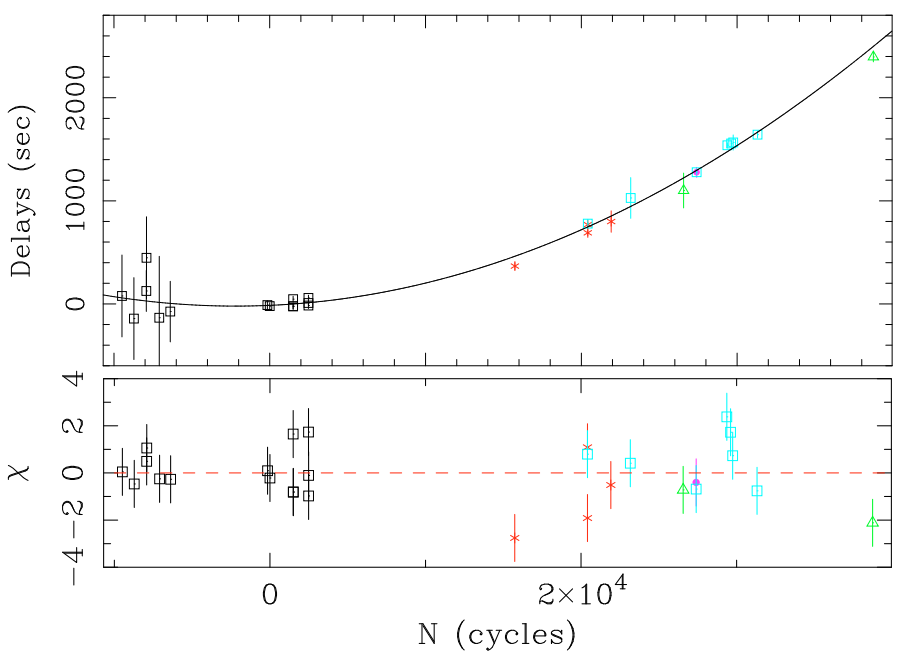

Fig. 1. Eclipse time delays with respect to a constant orbital period model plotted vs. the orbital cycle for all the available eclipse time measures spanning the period from 1977 to 2008 together with the best-fit parabola (top panel), and residuals in units of $\sigma$ with respect to the best-fit parabola (bottom panel). Different symbols indicate different datasets: black squares are from Hellier et al. (1990), red stars are from Parmar et al. (2000), cyan squares are from RXTE data, the magenta dot is from XMM-Newton data, and the green triangles are from Chandra data.

Table 2. Best-fit orbital solution for 2A 1822-371 derived from the analysis of the eclipse arrival times from 1977 to 2008.

\begin{tabular}{llrr}
\hline \hline Parameter & Units & P2000 & This work \\
\hline$T_{0}^{\mathrm{e}}$ & MJD & $45614.80964(15)$ & $45614.80948(14)$ \\
$P_{\text {orb } 0}$ & $\mathrm{~s}$ & $20054.1990(43)$ & $20054.2056(22)$ \\
$\dot{P}_{\text {orb }}$ & $10^{-10} \mathrm{~s} / \mathrm{s}$ & $1.78(20)$ & $1.499(71)$ \\
$\chi^{2} /$ d.o.f. & & $21.4 / 16$ & $38.69 / 25$ \\
\hline
\end{tabular}

Notes. Errors are at $1 \sigma$ c.l. on the last 2 digits. The value of $P_{\text {orb } 0}$ is referred to $T_{0}^{\mathrm{e}}$. The best-fit orbital parameters reported in this work are compared with the ephemeris given by P2000 (Parmar et al. 2000).

where the correction to the adopted value of the eclipse time, $\delta T_{0}^{\mathrm{e}}$, and to the adopted value of the orbital period, $\delta P_{\text {orb } 0}$, and the orbital period derivative, $\dot{P}_{\text {orb }}$, are the fit parameters. We get a very good fit with a $\chi^{2} /$ d.o.f. $=38.69 / 25=1.5$. In agreement with previous results, we find a highly significant derivative of the orbital period, which indicates that the orbital period in this system is increasing at a rate of $\dot{P}_{\text {orb }}=(1.499 \pm 0.071) \times 10^{-10} \mathrm{~s} / \mathrm{s}$. The best-fit values for the orbital parameters, calculated with the corrections we found from the fit of the parabolic trend of the eclipse epochs with Eq. (1), are shown in Table 2. Note that a similar orbital period derivative was recently found with new measures of optical eclipses by Bayless et al. (2009).

\section{Orbital evolution of 2A 1822-371}

Apart from mass transfer between the companion and the neutron star, the orbital evolution of this binary system is expected to be driven by the emission of gravitational waves and by magnetic braking. Under the further assumption of conservative mass transfer, orbital evolution calculations show that the orbital period derivative should be

$$
\begin{aligned}
\dot{P}_{\text {orb }}= & -3.0 \times 10^{-14} m_{1} m_{2,0.1} m^{-1 / 3} P_{5 \mathrm{~h}}^{-5 / 3} \times\left[1.0+T_{\mathrm{MB}}\right] \\
& \times\left[(n-1 / 3) /\left(n+5 / 3-0.2 m_{2,0.1} m_{1}^{-1}\right)\right] \mathrm{s} \mathrm{s}^{-1}
\end{aligned}
$$

(see Di Salvo et al. 2008; Verbunt 1993; see also Rappaport et al. 1987), where $m_{1}$ and $m$ are the mass of the primary, $M_{1}$, and the total mass, $M_{1}+M_{2}$, in units of $M_{\odot}$ respectively, $m_{2,0.1}$ is the mass of the secondary in units of $0.1 M_{\odot}, P_{5 \mathrm{~h}}$ is the orbital period in units of $5 \mathrm{~h}$ (that is appropriate for 2A 1822-371 because $\left.P_{\text {orb }}=5.57 \mathrm{~h}\right), n$ is the index of the mass-radius relation of the secondary $R_{2} \propto M_{2}^{\mathrm{n}}$, and where the term $T_{\mathrm{MB}} \sim 20$ takes into account the effect of the magnetic braking.

In line with Verbunt \& Zwaan (1981), Verbunt (1993), and King (1988; see Tauris 2001, for a review) we can parametrise this term as

$T_{\mathrm{MB}}=19.3\left(f / k_{0.277}\right)^{-2} m_{2,0.1}^{1 / 3} m_{1}^{-4 / 3} P_{5 h}^{2}$,

where $f$ is a dimensionless parameter of order of unity: preferred values are $f=0.73$ (Skumanich 1972) or $f=1.78$ (Smith 1979), and $k_{0.277}$ is the radius of gyration of the star $k$ in units of 0.277 , which is the appropriate value for a $1 M_{\odot}$ ZAMS star (Claret \& Gimenez 1989). Note that the expression for the MB term given in Verbunt (1993) is recovered from the above adopting $f=1$ and $k$ for a $1 M_{\odot}$ ZAMS star. Actually, Tauris (2001) discussed three different expression for the angular momentum losses due to $\mathrm{MB}, \dot{J}_{\mathrm{MB}}$, namely that proposed by Skumanich (Verbunt \& Zwaan 1981), that proposed by Stepien (1995), and that proposed by Rappaport et al. (1983), respectively. However $\left|\dot{J}_{\text {Stepien }}\right| \leq 0.1\left|\dot{J}_{\text {Skumanich }}\right|$ with $\left|\dot{J}_{\text {Rappaport }}\right|$ somewhat between them. Because we found that to describe 2A $1822-371$ a quite large $\dot{J}_{\mathrm{MB}}$ is required, we decided to adopt $\left|\dot{J}_{\text {Skumanich }}\right|$ which resulted in the term $T_{\mathrm{MB}} \sim 20$ adopted above.

The orbital period derivative we measured cannot be explained by a conservative scenario however. A positive orbital period derivative certainly indicates a mass-radius index $n<$ $1 / 3$; this is indeed a quite general result, which does not depend on the details of the angular momentum losses (see also Eq. (4) below). However, the orbital period derivative we measured, $\dot{P}_{\text {orb }}=1.50(7) \times 10^{-10} \mathrm{~s} / \mathrm{s}$, is about three orders of magnitude larger than what is expected even including the (strongest) MB term! This discrepancy is embarrassingly large suggesting that the conservative evolutionary scenario cannot be applied in this case. A similar conclusion was reached by Bayless et al. (2009), who give an improved ephemeris for this source based on new optical eclipse measures; these authors also note that an extremely high mass accretion rate onto the neutron star, corresponding to about four times the Eddington limit, would be required to explain the observed large orbital period derivative, and conclude that much of the transferred mass must be lost from the system. Below we show how the orbital period derivative we measured can be used to constrain the mass transfer in the system, and how this strongly indicates that a large fraction of the mass which the companion tries to transfer to the neutron star is lost by the system.

The mass-loss rate from the secondary can be easily calculated as a function of the orbital period of the system and the measured orbital period derivative combining the third Kepler law, which must be always satisfied by the orbital parameters of the system, with the condition that in this persistent system the neutron star is accreting mass through Roche Lobe overflow. This means that the radius of the secondary follows the evolution of the secondary Roche Lobe radius: $\dot{R}_{L 2} / R_{L 2}=\dot{R}_{2} / R_{2}$, where for the secondary we adopted a mass-radius relation $R_{2} \propto M_{2}^{n}$ and for the radius of the secondary Roche Lobe we adopted the Paczyński (1971) approximation $R_{L 2}=2 / 3^{4 / 3}[q /(1+q)]^{1 / 3} a$, where $a$ is the orbital separation, which is valid for small mass ratios, $q=M_{2} / M_{1} \leq 0.8$. From these conditions it is possible to 
derive a relation between the mass-loss rate from the secondary and the orbital period derivative

$\dot{m}_{-8}=3.5 \times(3 n-1)^{-1} m_{2,0.1}\left(\frac{\dot{P}_{-10}}{P_{5 \mathrm{~h}}}\right)$,

where $\dot{m}_{-8}$ is the secondary mass derivative (negative since the secondary star looses mass) in units of $10^{-8} M_{\odot} \mathrm{yr}^{-1}$, and $\dot{P}_{-10}$ is the orbital period derivative in units of $10^{-10}$. We stress that in Eq. (4) an expression for the angular momentum losses mechanism that drives the evolution of the system (e.g. MB or GR) does not explicitly appear. This is quite relevant because at present there is no general consensus on the absolute strength of the MB term nor on its functional dependence on the other orbital parameters. Indeed the effects of the driving mechanism are implicitly considered through the orbital period derivative, which is a measured quantity in our case.

Equation (4) can be inverted to derive the mass-transfer timescale $\tau_{\dot{M}}=M_{2} /\left(-\dot{M}_{2}\right)$

$\tau_{\dot{M}}=2.86 \times(1-3 n)\left(P_{5 \mathrm{~h}} / \dot{P}_{-10}\right) \times 10^{6} \mathrm{yr}$.

On this short time-scale the response of the secondary star must be adiabatic. For $m_{2} \sim 0.3$ (see below) the envelope is convective and the appropriate index is $n=-1 / 3$, in agreement with the condition $n<1 / 3$ discussed above. With this value we find $\tau_{\dot{M}} \sim$ $4 \times 10^{6} \mathrm{yr}$ for $2 \mathrm{~A} 1822-371$. We note that the Eddington limit (in units of $10^{-8} M_{\odot} \mathrm{yr}^{-1}$ ) for accretion onto a neutron star is $\dot{m}_{\mathrm{E}-8}=1.54 R_{6}\left(m_{1}\right)$, where $R_{6}\left(m_{1}\right)$ is the neutron star radius in units of $10^{6} \mathrm{~cm}$, which slightly depends on the neutron star mass once an equation of state (EoS) for the ultradense matter is adopted.

Thus, adopting $m_{2,0.1} \geq 3.3$ (Jonker et al. 2003) and $n=$ $-1 / 3$ in Eq. (4), we have to conclude that the secondary mass loss rate in 2A 1822-371 is super-Eddington. We are therefore forced to conclude that the evolution of the system is highly nonconservative.

In order to search for a possible evolutionary scenario for 2A 1822-371 we make the assumption that the neutron star is accreting at the maximum possible rate, i.e. the Eddington limit. It has to be noted that the Eddington limit strictly holds for a spherical geometry, and may not be a constraint for highly magnetised neutron stars for which the accreting matter is channeled onto the magnetic polar caps and the geometry of the matter distribution over the Alfvén surface may not be symmetric (see e.g. Basko \& Sunyaev 1976). However, our assumption is justified because the luminosity function for highly magnetized neutron stars (usually found in High Mass X-ray Binaries) does not disagree with this assumption (see e.g. Grimm et al. 2002). In particular, no highly magnetized neutron star is known to accrete at a rate much higher than the Eddington limit, and the most luminous high mass X-ray binaries containing a neutron star in our Galaxy reach luminosities of the order of the Eddington limit. Moreover, the extrapolated X-ray luminosity of 2A 1822-371 does not indicate an extremely high X-ray luminosity. Hence we do not have any evidence that the limiting mass accretion rate in this source is very different from the Eddington limit.

This results in the following condition:

$-\beta \times \dot{m}_{-8}=1.54 R_{6}\left(m_{1}\right)$,

where $\beta$ is the fraction of the mass lost by the secondary, which is accreted by the neutron star, namely $\dot{M}_{1}=-\beta \dot{M}_{2}$, where $\dot{M}_{1}$ and $\dot{M}_{2}$ are the mass derivatives of the primary and the secondary, respectively. We consider two EoS, namely the moderately soft FPS and the stiffer L (Cook et al. 1994), which give the relation

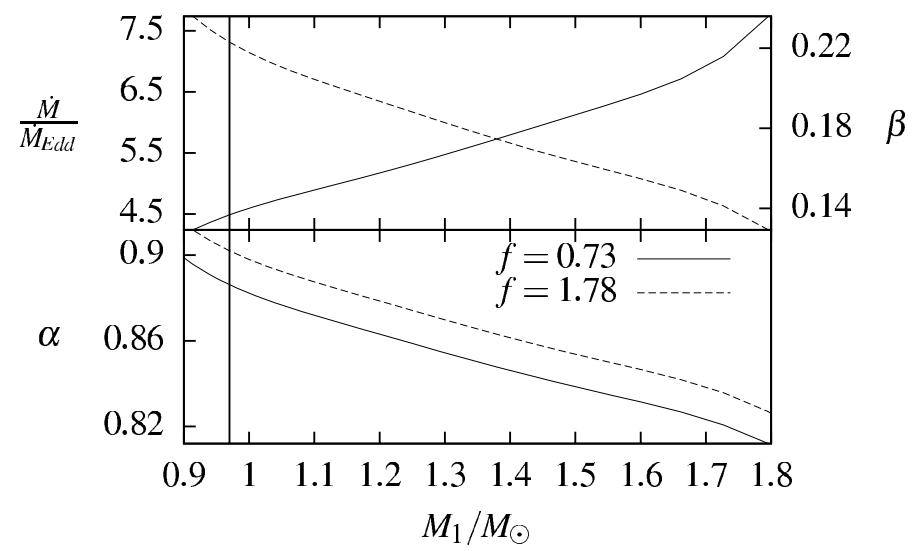

Fig. 2. Secondary mass loss rate in units of the Eddington limit for the FPS EoS (top panel, solid line), fraction $\beta$ of the mass lost by the secondary star which is accreted onto the neutron star (top panel, dashed line), and specific angular momentum of the mass leaving the system, $\alpha$, in units of the specific angular momentum of the secondary computed for two different prescription of the magnetic braking strength, namely $f=0.73$ (Skumanich 1972) and $f=1.78$ (Smith 1979) (bottom panel). The vertical line at $0.97 M_{\odot}$ represents the lower limit of the neutron star mass derived by Jonker et al. (2003).

$R_{6}\left(m_{1}\right)$ for the neutron star radius. With this and considering the mass function of the system derived from the timing analysis of the neutron star spin (Jonker \& van der Klis 2001), Eqs. (4) and (6) can be solved to derive $\dot{M}_{2}$ and $\beta$ for any value of $m_{1}$ between $m_{1 \mathrm{MIN}}=0.97$ and $m_{1 \mathrm{MAX}}$, which depends on the particular EoS adopted, which is 1.8 and 2.5 for EoS FPS and L, respectively. In Fig. $2 \dot{M}_{2}$, in units of the Eddington mass transfer rate, and $\beta$ are plotted for the appropriate range of neutron star masses for the FPS case (for the L EoS the value of $\beta$ is $30 \%$ higher, while $\dot{M}_{2}$ in units of the Eddington limit is $30 \%$ lower).

We now consider Eq. (3) of Di Salvo et al. (2008), which expresses the conservation of the angular momentum of the system

$\frac{\dot{P}_{\text {orb }}}{P_{\text {orb }}}=3\left[\frac{j}{J_{\text {orb }}}-\frac{\dot{M}_{2}}{M_{2}} g(\beta, q, \alpha)\right]$,

where, in this case,

$\frac{\dot{J}}{J_{\text {orb }}}=-5.5 \times 10^{-19}\left[1.0+T_{\mathrm{MB}}\right] m_{1} m_{2,0.1} m^{-1 / 3} P_{5 h}^{-8 / 3}$

represents all the possible losses of angular momentum from the system caused by MB and GR, where $T_{\mathrm{MB}}$ is given by Eq. (3) and

$g(\beta, q, \alpha)=1-\beta q-(1-\beta)(\alpha+q / 3) /(1+q)$

takes into account the effects of angular momentum losses because of mass loss from the system. $\alpha$ is the specific angular momentum of the mass leaving the system, $l_{\mathrm{ej}}$, in units of the specific angular momentum of the secondary, that is: $\alpha=$ $l_{\mathrm{ej}} /\left(\Omega_{\mathrm{orb}} r_{2}^{2}\right)=l_{\mathrm{ej}} P_{\mathrm{orb}}\left(M_{1}+M_{2}\right)^{2} /\left(2 \pi a^{2} M_{1}^{2}\right)$, where $r_{2}$ is the distance of the secondary star from the center of mass of the system, and $a$ is the orbital separation.

Adopting the two values of $f$ discussed above, namely $f=$ 0.73 (Skumanich 1972) or $f=1.78$ (Smith 1979), and $k_{0.277}=1$, Eqs. (3), (7), (8), and (9) can be solved to derive $\alpha$ as a function of $m_{1}$. In Fig. $2 \alpha$ is plotted for the appropriate range of neutron star masses for the FPS case (for the L EoS the values of $\alpha$ are $7 \%$ higher). 
The values of $\alpha$ we obtain are in between the specific angular momentum at the inner Lagrangian point, $\alpha_{\mathrm{L} 1}=[1-0.462(1+$ $\left.q)^{2 / 3} q^{1 / 3}\right]^{2} \sim 0.4$ for $2 \mathrm{~A} 1822-371$, and the specific angular momentum of the secondary, $\alpha_{2}=1.0$, and actually quite close to $\alpha_{2}$. This is expected if the mass lost by the secondary star is blown away because of the radiation pressure exerted by the Eddington luminosity generated by the accretion onto the neutron star.

For both the adopted EoS and all the possible values of the neutron star mass, the values of $\beta$ are in the range $0.13-0.29$, which means that the mass transfer in 2A 1822-371 is not conservative, at least, at $70 \%$ level, which, as we already noted, is true independently of any assumption on the particular angular momentum losses. Interestingly, this is the key that opens the possibility of constructing a consistent secular evolution for this system. Indeed the contact condition, $\dot{R}_{L 2} / R_{L 2}=\dot{R}_{2} / R_{2}$, can be solved to derive a theoretical prediction for the mass-loss rate once a prescription is given for the possible losses of angular momentum from the system caused by MB and GR as in Eq. (8):

$$
\begin{aligned}
\dot{m}_{-8}= & -3.5 \times 10^{-4}\left[1.0+T_{\mathrm{MB}}\right] m_{1} m_{2,0.1}^{2} m^{-1 / 3} P_{5 h}^{-8 / 3} \\
& \times F(n, g(\beta, q, \alpha)),
\end{aligned}
$$

where

$F(n, g(\beta, q, \alpha))=[n-1 / 3+2 g(\beta, q, \alpha)]^{-1}$.

The function $F(n, g(\beta, q, \alpha))$ is very sensitive to the scenario adopted: for a conservative scenario $(\beta=1) F(n, g(\beta, q, \alpha)) \sim$ 1.5 while for $\beta$ in the range $0.13-0.22$ (which is appropriate for the FPS EoS and all the possible values of the neutron star mass) $F(n, g(\beta, q, \alpha)) \sim 40$. This means that the term acting to shrink the secondary Roche Lobe - because of the extra angular momentum losses caused by the mass expelled from the system - determines an amplification of the mass-loss rate through the function $F(n, g(\beta, q, \alpha))$ with respect to a fully conservative case. This amplified mass-loss rate in turn determines the high value of the orbital period derivative observed in this system. Consequential angular momentum losses (CAML), i.e. angular momentum losses that are themselves the result of mass transfer, have been proposed in the context of cataclysmic variables (CVs) evolution (see e.g. King \& Kolb 1995).

Inserting the values determined in this paper for $2 \mathrm{~A} 1822$ 371 in Eq. (5) we find $\tau_{\dot{M}}=2.1 \times 10^{6} \mathrm{yr}$. This means that the system as it is observed now will probably end on this timescale, possibly with the tidal disruption of the companion star. Indeed, King \& Kolb (1995) argued that mass transfer could be unstable when CAML are present. This time-scale is extremely short, which indicates that it is possible that some short orbital period
LMXBs can last much shorter than what was previously thought. This evolutionary phase, characterised by a super-Eddington mass transfer rate, may be a common phase in the evolution of LMXBs, albeit short-living. Because this phase should not last more than a few million years, there may be very few observed systems in this phase (e.g. the so-called Z-sources, which are persistently bright LMXBs). This could have profound implications for the estimate of the actual number of LMXBs produced in the Galaxy as inferred from the observed ones, and also for the predicted number of millisecond binary pulsar. We note that this would help to bring the number of LMXBs in line with the estimated number of millisecond binary pulsars. But a detailed analysis of this delicate and long-standing problem needs a dedicated study of this almost unstable phase of the orbital evolution, which is beyond the scope of this paper and will be discussed in a forthcoming paper.

Acknowledgements. This work is supported by the Italian Space Agency, ASI-INAF I/088/06/0 contract for High Energy Astrophysics. We thank the referee for useful comments on the manuscript.

\section{References}

Basko, M. M., \& Sunyaev, R. A. 1976, MNRAS, 175, 395

Bayless, A. J., Robinson, E. L., Hynes, R. I., Ashcraft, T. A., \& Cornell, M. E. 2010, ApJ, 709, 251

Claret, A., \& Gimenez, A. 1989, A\&AS, 81, 37

Cook, G. B., Shapiro, S. L., \& Teukolsky, S. A. 1994, ApJ, 424, 823

Di Salvo, T., Burderi, L., Riggio, A., Papitto, A., \& Menna, M. T. 2008, MNRAS, 389,1851

Grimm, H. J., Gilfanov, M., \& Sunyaev, R. 2002, A\&A, 391, 923

Hellier, C., \& Mason, K. O. 1989, MNRAS, 239, 715

Hellier, C., Mason, K. O., Smale, A. P., \& Kilkenny, D. 1990, MNRAS, 244, 39

Iaria, R., Di Salvo, T., Burderi, L., \& Robba, N. R. 2001, ApJ, 557, 24

Jonker, P. G., \& van der Klis, M. 2001, ApJ, 553, L43

Jonker, P. G., van der Klis, M., \& Groot, P. 2003, MNRAS, 339, 663

King, A. R. 1988, QJRAS, 29, 1

King, A. R., \& Kolb, U. 1995, ApJ, 439, 330

Mason, K. O., \& Cordova, F. A. 1982, ApJ, 262, 253

Paczyński, B. 1971, ARA\&A, 9, 183

Parmar, A. N., Oosterbroek, T., Del Sordo, S., et al. 2000, A\&A, 356, 175

Rappaport, S., Ma, C. P., Joss, P. C., \& Nelson, L. A. 1987, ApJ, 322, 842

Rappaport, S., Verbunt, F., \& Joss, P. C. 1983, ApJ, 275, 713

Skumanich, A. 1972, ApJ, 171, 565

Smith, M. A. 1979, PASP, 91, 737

Stepien, K. 1995, MNRAS, 274, 1019

Tauris, T. M. 2001, ASP Conf. Ser., 229, 145

van Paradijs, J., \& McClintock, J. E. 1994, A\&A, 290, 133

Verbunt, F. 1993, ARA\&A, 31, 93

Verbunt, F., \& Zwaan, C. 1981, A\&A, 100, L7

White, N. E., Becker, R. H., Boldt, E. A., et al. 1981, ApJ, 247, 994 